\title{
Coupling killing to neutralization: combined therapy with ceftriaxone/Pep19-2.5 counteracts sepsis in rabbits
}

\author{
Sergio Bárcena-Varela ${ }^{1}$, Guillermo Martínez-de-Tejada ${ }^{1}$, Lukas Martin ${ }^{2}$, Tobias Schuerholz ${ }^{3}$, \\ Ana Gloria Gil-Royo ${ }^{4}$, Satoshi Fukuoka ${ }^{5}$, Torsten Goldmann ${ }^{6,7,8}$, Daniel Droemann ${ }^{8,9}$, Wilmar Correa ${ }^{10}$, \\ Thomas Gutsmann ${ }^{10}$, Klaus Brandenburg ${ }^{10}$ and Lena Heinbockel ${ }^{10}$
}

Sepsis, which is induced by severe bacterial infections, is a major cause of death worldwide, and therapies combating the disease are urgently needed. Because many drugs have failed in clinical trials despite their efficacy in mouse models, the development of reliable animal models of sepsis is in great demand. Several studies have suggested that rabbits reflect sepsisrelated symptoms more accurately than mice. In this study, we evaluated a rabbit model of acute sepsis caused by the intravenous inoculation of Salmonella enterica. The model reproduces numerous symptoms characteristic of human sepsis including hyperlactatemia, hyperglycemia, leukopenia, hypothermia and the hyperproduction of several pro-inflammatory cytokines. Hence, it was chosen to investigate the proposed ability of Pep19-2.5-an anti-endotoxic peptide with high affinity to lipopolysaccharide and lipoprotein-to attenuate sepsis-associated pathologies in combination with an antibiotic (ceftriaxone). We demonstrate that a combination of Pep19-2.5 and ceftriaxone administered intravenously to the rabbits (1) kills bacteria and eliminates bacteremia 30 min post challenge; (2) inhibits Toll-like receptor 4 agonists in serum 90 min post challenge; (3) reduces serum levels of pro-inflammatory cytokines (interleukin-6 and tumor necrosis factor $\alpha$ ); and (4) reverts to hypothermia and gives rise to temperature values indistinguishable from basal levels $330 \mathrm{~min}$ post challenge. The two components of the combination displayed synergism in some of these activities, and Pep19-2.5 notably counteracted the endotoxin-inducing potential of ceftriaxone. Thus, the combination therapy of Pep19-2.5 and ceftriaxone holds promise as a candidate for human sepsis therapy.

Experimental \& Molecular Medicine (2017) 49, e345; doi:10.1038/emm.2017.75; published online 16 June 2017

\section{INTRODUCTION}

Sepsis is one of the leading causes of death in intensive care units worldwide, with mortality rates ranging from $10 \%$ to over $40 \%$ depending on the clinical associations. ${ }^{1}$ Triggered by an infection and characterized by an inflammatory state affecting the patient's whole body, sepsis accounts for 300-1000 cases per 100000 persons in the United States. ${ }^{2}$ Thus, the urgency for an effective therapy against sepsis cannot be overemphasized.

The established animal models of sepsis differ in their response to the triggering factor of inflammation and consequently in their reaction to medications. This may partly explain the failure of multiple clinical trials investigating drug strategies for sepsis therapy during recent years. Moreover, the difficulties encountered are likely due to major differences in immune system functioning between animal species and humans. ${ }^{3}$ Animal sepsis models widely vary and include intraperitoneal (i.p.)/intravenous (i.v.) injection of lipopolysaccharide (LPS) or dead bacteria (endotoxemia), inoculation of live bacteria or specific surgeries such as cecal ligation and puncture, colon ascendens stent peritonitis and polymicrobial

\footnotetext{
${ }^{1}$ Department of Microbiology and Parasitology, Universidad de Navarra, Pamplona, Spain; ${ }^{2}$ Department of Intensive Care and Intermediate Care, University Hospital Aachen, Aachen, Germany; ${ }^{3}$ Department of Anaesthesia and Intensive Care, University of Rostock, Rostock, Germany; ${ }^{4}$ Department of Pharmacology and Toxicology, Universidad de Navarra, Pamplona, Spain; ${ }^{5}$ National Institute of Advanced Industrial Science and Technology AIST, Takamatsu, Japan; ${ }^{6}$ Department of Pathology, University Hospital of Luebeck, Luebeck, Germany; ${ }^{7}$ Department of Pathology, Leibniz Research Center, Borstel, Germany; ${ }^{8}$ Airway Research Center North (ARCN), Member of the German Center for Lung Research (DZL), Großhansdorf, Germany; ${ }^{9}$ Medical Clinic III, Pulmonology/Infectious Diseases, University Hospital Schleswig-Holstein, Luebeck, Germany and ${ }^{10}$ Division of Biophysics, Research Center Borstel, Leibniz-Center for Medicine and Biosciences, Borstel, Germany

Correspondence: Professor K Brandenburg, Division of Biophysics, Research Center Borstel, Leibniz-Center for Medicine and Biosciences, Parkallee 10, Borstel D-23845, Germany.

E-mail: kbrandenburg@fz-borstel.de

Received 29 October 2016; revised 5 January 2017; accepted 23 January 2017
} 
peritoneal contamination and infection. ${ }^{4}$ Although surgical models are known to reflect more accurately the clinical scenario, they usually require a long-term evaluation, and their mortality rates vary depending on surgical efficiency. On the other side, non-surgical models (for example, inoculation of cells or antigens into the animal) are widely used for acute sepsis evaluation, being highly controlled and less laborious than models relying on the animal microbiota as the infectious agent. ${ }^{5}$

Furthermore, most tested therapies aim at killing the bacteria or modulating the immune response. Yet, these treatments do not address the major underlying cause of sepsis- that is, the release of toxins (pathogenicity factors) by the bacteria. ${ }^{6}$ Thus, besides the standard supportive intensive care treatment, no satisfactory specific therapeutic option exists to date. Current therapies with antibiotics aim at killing bacteria, but this frequently leads to the release of the pathogenicity factors, hence aggravating the patient's inflammatory response. ${ }^{7}$ Considering the rapid increase in multiresistant strains and the lack of newly approved antibiotics, the situation of the most severely ill patients in intensive care units becomes more and more threatening.

One approach alternative to conventional antibiotic-based therapy entails the use of antimicrobial peptides (AMPs). Until now, there are only a limited number of approved AMP drugs available, although daptomycin ${ }^{8}$ (against skin and skin structure infections) and colistin (against multiresistant Gram-negative bacteria) are two relevant agents. Thus, the recent development of a synthetic anti-LPS peptide, Pep19-2.5 (Aspidasept), appears promising also for a broader application. ${ }^{9}$ Recently, it has been demonstrated that this compound has high affinity not only for Gram-negative LPS but also for Gram-positive lipoprotein (LP) in various in vitro and in vivo mouse experiments. ${ }^{10}$ Pep19-2.5 has also been shown to be protective in a murine model of septicemia as well as in cecal ligation and puncture-challenge, causing a considerable decrease in inflammation that correlated with a survival benefit of the animals. ${ }^{10,11}$ Mouse models have drawbacks with regard to the situation in human sepsis patients and should therefore be complemented with studies in more relevant animals (rabbits or pigs) after initial testing in the practical model. ${ }^{12}$ One of the most prominent differences between mouse and man is the relative insensitivity of mice with respect to the induction of inflammation, particularly when LPS is used as an inducer. In fact, along with LPS, animals must be co-administered with galactosamine to sensitize them to endotoxin and cause a pro-inflammatory response similar to that of humans. ${ }^{13,14}$ The dose $\left(\mathrm{LD}_{50}\right)$ values of LPS inducing severe illness and hypotension in mice and rats are 1000- to 10000 -fold higher, respectively, than in humans. ${ }^{15}$ By contrast, rabbits are considerably more responsive to LPS, and results from preclinical studies in this non-rodent species are expected to better predict the results of clinical trials. ${ }^{16}$

The aim of this study was to use a novel rabbit model of sepsis to evaluate the tolerability and therapeutic efficacy of a drug combination that was previously shown to be protective in mice. In this model, bacteremia was induced in the animals by injecting live cells of Gram-negative (Salmonella) bacteria. The combined treatment of the antibiotic ceftriaxone to kill the bacteria and Pep19-2.5 to neutralize released LPS was evaluated. The data showed that this combination is able to drastically reduce the strong inflammation as evidenced by a drop in the levels of interleukin 6 (IL-6) and tumor-necrosis-factor $\alpha$ (TNF $\alpha)$. Endotoxin detection in the blood of the animals showed that this decrease in cytokine concentrations is directly correlated with a marked reduction of the detectable LPS concentration in serum. Essentially, we demonstrate that Pep19-2.5 and ceftriaxone work in conjunction to kill bacteria and reduce inflammation.

\section{MATERIALS AND METHODS}

\section{Bacterial strains and culture conditions}

Salmonella enterica serovar Minnesota (SF1114, smooth form) was grown in tryptic soy broth (TSB; Difco Laboratories, Detroit, MI, USA) at $37^{\circ} \mathrm{C}$ with orbital shaking. For inoculum preparation, an overnight liquid culture was centrifuged $(4000 \mathrm{~g}, 5 \mathrm{~min}$ ), and the pellet was suspended in sterile saline and adjusted to $\mathrm{OD}_{600 \mathrm{~nm}}=1.0$, approximately corresponding to $3.7 \times 10^{9}$ colony-forming units (CFUs) per $\mathrm{ml}$. For viable cell counting, aliquots were plated onto TSB agar and were incubated for $24 \mathrm{~h}$.

\section{Peptide synthesis}

The synthesis and purification of Pep19-2.5 (Lot 1053821) was previously described, ${ }^{17}$ and the batch used in the present work was produced under GMP conditions by BACHEM (Bubendorf; Switzerland). The purity was $>95 \%$, and the amino-acid sequence of this $20^{\prime}$ mer is GCKKYRRFRWKFKGKFWFWG, containing a C-terminal amidation.

\section{Animal experiments}

All experiments were approved by the Animal Ethical Committee of the University of Navarra (protocol number: 182-12). Care and handling of the animals were in accordance with the ICH and OECD guidelines. Male New Zealand rabbits $(2-2.5 \mathrm{~kg}$ in weight; Granja San Bernardo S.I., Tulebras, Spain) were housed under standard conditions with free access to food and water. The animals were granted a 7-day acclimatization period prior to experimentation, and they were food fasted $12-14 \mathrm{~h}$ before inoculation. Each day, three animals were challenged, treated and killed. The inoculum consisted of $1 \mathrm{ml}$ of sterile saline approximately containing $3.7 \times 10^{9} \mathrm{CFU}$ of $S$. enterica serovar Minnesota (Supplementary Figure S1). Animals ( $n=6$ per experimental group) were intravenously (i.v.) inoculated by the marginal ear vein. Treatments were administered i.v. with a total volume of $2 \mathrm{ml}$ immediately after the inoculum by the marginal ear and consisted of ceftriaxone ( $15 \mathrm{mg} \mathrm{kg}^{-1}$; Sigma-Aldrich, Madrid, Spain) dissolved in saline and/or Pep19-2.5 (5 $\left.\mathrm{mg} \mathrm{kg}^{-1}\right)$ dissolved in saline. A group of animals was left untreated and received only vehicle. Blood samples were extracted from the marginal ear vein at different time points post challenge (0, 2, 30, 90 and $180 \mathrm{~min})$. Fifteen minutes before each blood extraction, acepromazine $\left(1 \mathrm{mg} \mathrm{kg}^{-1}\right.$; Sigma-Aldrich) was i.v. administered for sedative and vasodilator purposes. All the procedures were performed aseptically and using sterile labware. The rectal temperature was monitored at 0, 90, 150, 210 and $330 \mathrm{~min}$ post challenge. Six hours after inoculation, the 
animals were killed by the administration of a lethal dose of T-61 (Sigma-Aldrich)

\section{Blood and serum testing}

For bacteremia analysis, whole blood was serially diluted (1:10 in sterile saline) and plated in TSB Agar plates. Viable counts were determined after $16-18 \mathrm{~h}$ of incubation at $37^{\circ} \mathrm{C}$. For serum preparation, blood samples $(4 \mathrm{ml})$ were taken under sterile conditions, centrifuged (3000 r.p.m., $10 \mathrm{~min}, 4^{\circ} \mathrm{C}$ ) using serum-separating tubes and stored at $-80^{\circ} \mathrm{C}$ for subsequent analysis. Fluoride/EDTA and EDTA-containing vacutainer tubes were used for lactate quantification and hematological analysis, respectively (Sysmex XT-1800i; Sant Just Desvern, Spain). Biochemical parameters were determined using a Hitachi-911 automatic analyzer (Hitachi Medical Systems, Madrid, Spain).

\section{Post-mortem analyses}

Post-mortem samples of the spleen, liver, kidneys and lungs were extracted and weighed, and $1 \mathrm{~g}$ of each was homogenized and serially diluted for plating and viable counting. The remainder of the organ was fixed by immersion in $4 \%$ formaldehyde and was subsequently prepared for inclusion, cutting and hematoxylin-eosin staining. All the procedures were performed aseptically and using sterile labware. Anatomopathological analyses of histological preparations were carried out using the Anatomopathologic Diagnostic Service for Laboratory Animals (University of Zaragoza, Spain).

\section{Endotoxin and cytokine quantification}

Endotoxin was indirectly quantified by measuring IL-8 in a Toll-like receptor (TLR)4-expressing cell line that responds to LPS by secreting this cytokine (HEK-293/TLR4; kindly provided by Dr Juan José Lasarte). To account for the presence of potential IL-8 inducing compounds other than LPS in rabbit serum, IL-8 levels were measured in an identical cell line lacking the TLR4 receptor (HEK-293/LacZ). Cells were cultured in Dulbecco's modified Eagle's medium supplemented with $4.5 \mathrm{~g} \mathrm{l}^{-1}$ glucose, $10 \%$ heat-inactivated fetal bovine serum, penicillin/streptomycin and blasticidin $\left(5 \mu \mathrm{g} \mathrm{ml}^{-1}\right.$ for each; GIBCO; Thermo Fisher Scientific, Alcobendas, Spain) at $37^{\circ} \mathrm{C}$ in $5 \% \mathrm{CO}_{2}$. In the case of HEK-293/TLR4, cells were supplemented with $25 \mu \mathrm{g} \mathrm{ml}^{-1}$ hygromycin B (Invivogen Ultrapure; InvivoGen, San Diego CA, USA). Cells $\left(5 \times 10^{5}\right)$ were exposed to rabbit serum (1:10 dilution) for $24 \mathrm{~h}$, and IL- 8 was then quantified in the supernatants by solid-phase sandwich enzyme-linked immunosorbent assay (R\&D Systems; Madrid, Spain). Assays were performed in triplicate. The serum levels of IL- 6 and TNF $\alpha$ were determined using solid-phase sandwich enzyme-linked immunosorbent assay (R\&D Systems) following the manufacturer's instructions.

\section{Statistics}

The data were statistically analyzed using SPSS V15.0 software (IBM España, Madrid, Spain). analysis of variance was applied to study the potential differences in the bacterial load, endotoxin levels, cytokine concentrations and biochemical parameters. If there was homogeneity of variances, parametric Student-Neuman-Keuls post hoc test was conducted. Otherwise, the Tamhane test was applied. For the group comparisons of temperatures at different time points, repeated measures factorial design-analysis of variance was applied, followed by Student's $t$-test for related measures, adjusting the average s.d. Graphics were prepared using GraphPad Prism V6.01 software (GraphPad Software, La Jolla, CA, USA).

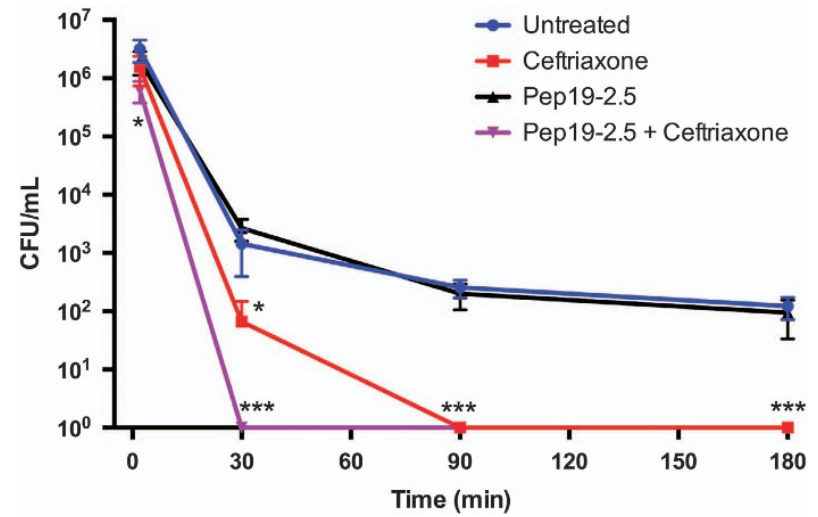

Figure 1 Kinetics of the bacteremia in rabbits infected with $S$. enterica and subsequently treated with Pep19-2.5 and/or ceftriaxone. At time 0 , animals ( $n=6$ per group) received an i.v. injection containing $3.7 \times 10^{9}$ CFU of $S$. enterica serovar Minnesota. Immediately afterwards, animals were treated with ceftriaxone (15 mg kg-1; i.v.) and/or Pep19-2.5 (5 mg kg-1; i.v.). A group of animals was left untreated and received only vehicle (saline). At the indicated time points, whole blood was extracted, serially diluted and plated for viable counting. Differences were statistically analyzed using analysis of variance followed by post hoc tests $\left({ }^{*} P<0.05 ;{ }^{* *} P<0.01 ;{ }^{* *} P<0.001\right)$.

\section{RESULTS}

\section{Kinetics of bacteremia}

To evaluate whether the combined administration of Pep19-2.5 and ceftriaxone reduces the bacterial burden in rabbits, we intravenously inoculated a group of New Zealand rabbits with $\sim 3.7 \times 10^{9} \mathrm{CFU}$ of $S$. enterica. Immediately after inoculation, the animals received a single i.v. administration of Pep19-2.5 and ceftriaxone. As a control, we treated the animals with either one of those therapies, whereas a third group received only vehicle (saline). In addition, to verify that inocula prepared in different days had a comparable size, we plated each bacterial suspension on solid medium and performed viable counts (Supplementary Figure S1). Interestingly, at the earliest time point ( $2 \mathrm{~min}$ post challenge), the combined therapy was the only treatment capable of reducing bacteremia (Figure 1). The two-component therapy also outperformed the single ceftriaxone-based therapy $30 \mathrm{~min}$ post challenge because the former was the only treatment displaying sterilizing activity (Figure 1). By contrast, Pep19-2.5 by itself displayed no detectable antibacterial activity at any time point.

\section{Endotoxin levels in rabbit serum}

To investigate whether any of the treatments had LPS-neutralizing activity, we quantified endotoxin levels in rabbit serum $90 \mathrm{~min}$ post challenge. We first tried to directly measure LPS levels using an Limulus amebocyte lysate-based method, but this approach rendered poor and unreliable results, likely due to serum compounds interfering with the Limulus amebocyte lysate test. To circumvent this problem, we quantified endotoxin concentrations in serum using TLR4-expressing cells that respond to LPS by secreting IL-8, which was subsequently measured by enzyme-linked 


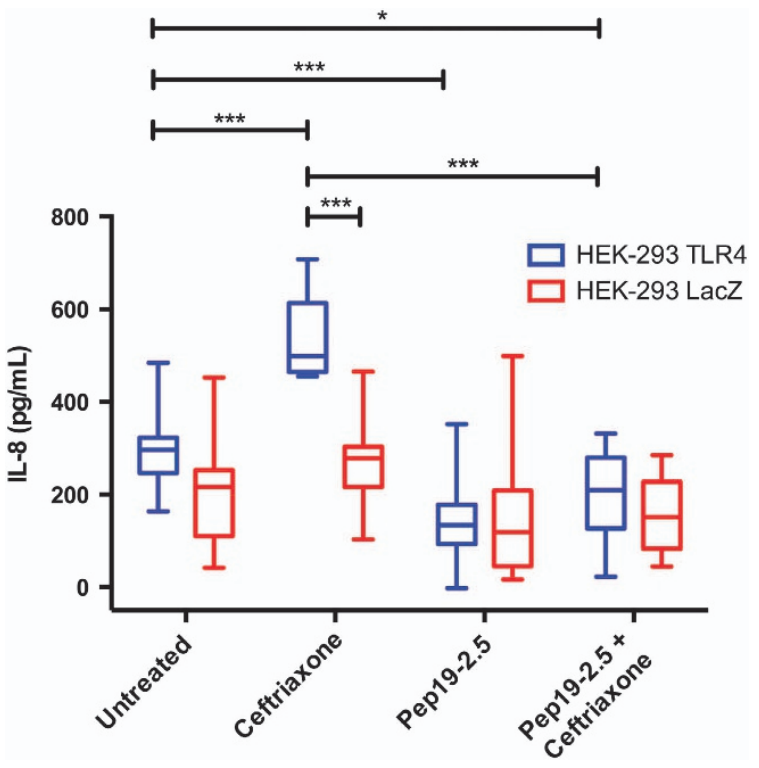

Figure 2 Quantification of the levels of TLR4 agonists in rabbit serum $90 \mathrm{~min}$ post challenge. The levels of TLR4 agonist (for example, LPS) were quantified in rabbit serum by measuring the production of IL-8 in TLR4-expressing cells (HEK-293/TLR4) incubated with serum samples ( $n=6$ per group). As a control to account for potential IL-8-inducing compounds other than LPS, a duplicate measurement was performed in an identical cell line lacking the TLR4 receptor (HEK-293/LacZ). Cells were exposed to rabbit serum for $24 \mathrm{~h}$, and IL-8 was quantified in the supernatants by solid-phase sandwich enzyme-linked immunosorbent assay. Assays were performed in triplicate, and the results were analyzed using analysis of variance followed by post hoc tests $\left({ }^{*} P<0.05 ;{ }^{*} P<0.01 ;{ }^{* *} P<0.001\right)$. The horizontal line within the boxes represents the median, whereas the lower part represents the 25th percentile, and the upper part represents the 75th percentiles. The whiskers represent the range of the values.

immunosorbent assay. In control experiments, we demonstrated that LPS quantitatively induced IL-8 secretion in these cells (data not shown). To account for the presence of potential IL-8-inducing compounds other than LPS in rabbit serum, IL-8 levels were measured in an identical cell line lacking the TLR4 receptor. As shown in Figure 2, the concentrations of TLR4 agonists in serum from ceftriaxone-treated rabbits were much higher than those measured in infected rabbits not treated with the antibiotic. This difference was not detectable in cells lacking TLR4. In marked contrast to the liberation of TLR4 agonists by ceftriaxone, Pep19-2.5 showed a potent ability to neutralize TLR4 agonists (Figure 2). Notably, rabbits treated with the combined therapy had very low serum levels of TLR4 agonists, demonstrating that Pep19-2.5 counteracts the LPS-releasing potential of ceftriaxone.

\section{Concentration of pro-inflammatory cytokines}

Based on the ceftriaxone-related release of TLR4 agonist, we next investigated the pro-inflammatory response of rabbits on S. enterica challenge. As shown in Figure 3, we measured the serum concentrations of TNF $\alpha$ and IL- 6 at $90 \mathrm{~min}$ and $180 \mathrm{~min}$ post challenge, respectively, coinciding with the peak concentration of each cytokine in serum, as determined in preliminary assays (Supplementary Figure S2). Ceftriaxone treatment displayed a trend to increase both the IL-6 and TNF $\alpha$ serum levels compared with untreated animals. By contrast, Pep19-2.5 not only lacked pro-inflammatory activity when given alone but also counteracted the IL-6- and TNFo-inducing ability of ceftriaxone. Notably, animals receiving the combined therapy had levels of IL- 6 even lower than those measured in untreated animals (Figure 3a), suggesting that the two components of the combination act in synergy to neutralize this cytokine.

\section{Metabolic parameters}

Abnormally high serum values of both glucose and lactate (that is, hyperglycemia and hyperlactatemia, respectively) are common during acute infection, particularly in patients with sepsis. To investigate whether our rabbit model reproduces these symptoms, we measured the serum levels of glucose and lactate throughout the course of the experiment. Inoculation of S. enterica caused a significant and progressive increase in glucose and lactate levels that was even more severe for the latter marker (Supplementary Figure S3). Neither the single nor combined treatment, however, was found to significantly reverse these increments at any time point (Supplementary Figure S3).

\section{Hematological markers}

Infections are known to induce leukocytosis, whereas leukopenia is characteristic of acute sepsis due to overwhelming infections. As shown in Supplementary Figure S4, the inoculation of $S$. enterica caused a severe decrease in white blood cells, which was of similar magnitude in both the untreated and treated groups ( $60 \%$ of the initial value).

\section{Temperature monitoring}

Sepsis in humans is known to induce either hypothermia or hyperthermia. To study whether any of these symptoms could be detected in our animal model, we monitored the rectal temperature of all the animals throughout the assay. As shown in Figure 4, the inoculation of S. enterica markedly reduced the rabbit temperature from $38^{\circ} \mathrm{C}$ at the beginning of the experiment to $\sim 35.5^{\circ} \mathrm{C}$ at the late time points post challenge (330 min). The kinetics of hypothermia in animals treated with Pep19-2.5 tended to be more rapid and to reach lower temperature values, but this trend was not significant. However, the apparent pro-inflammatory activity exhibited by ceftriaxone was not associated with aggravated hypothermia compared with untreated animals. Remarkably, when ceftriaxone was administered combined with Pep19-2.5, the two compounds acted jointly, leading to less-intense hypothermia (Figure 4). This convergent activity reverted hypothermia and resulted in a rise of the temperature $330 \mathrm{~min}$ after S. enterica challenge that was indistinguishable from basal levels (Figure 4). 


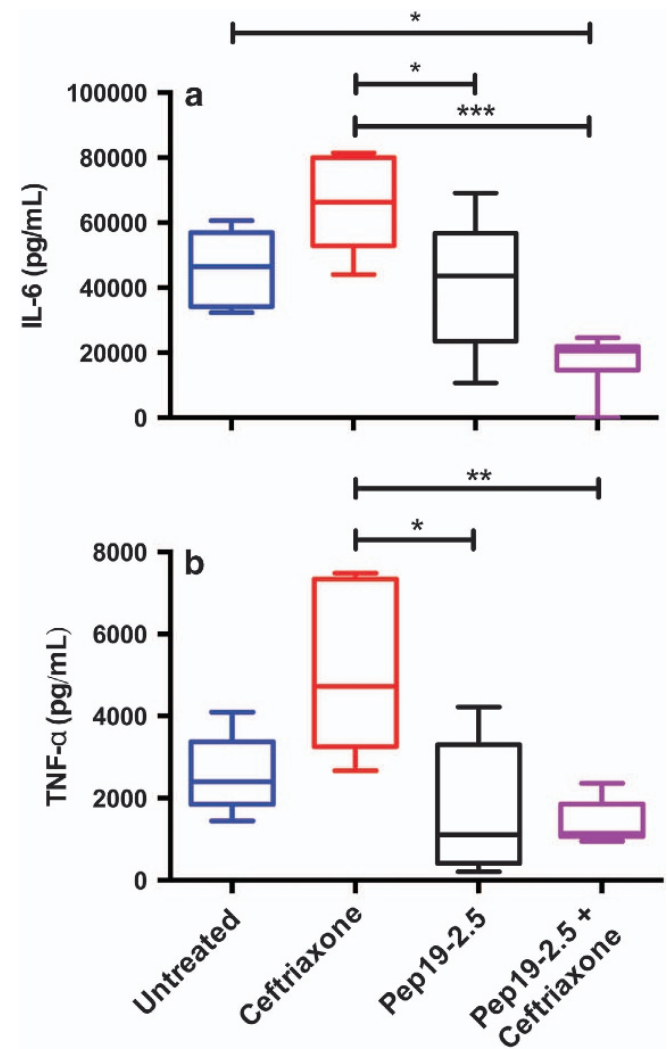

Figure 3 Quantification of the levels of IL-6 and TNF $\alpha$ in rabbit serum at different time points post challenge. IL- 6 (a) and TNF $\alpha$ (b) were quantified in animal sera ( $n=6$ per group) by solid-phase sandwich enzyme-linked immunosorbent assay at $180 \mathrm{~min}$ and 90 min post challenge, respectively, coinciding with the peak concentration of each cytokine (Supplementary Figure S2). Assays were performed in triplicate, and the results were analyzed using analysis of variance followed by post hoc tests $\left({ }^{*} P<0.05 ;{ }^{*} P<0.01 ; * * * P<0.001\right)$. The horizontal line within the boxes represents the median, whereas the lower part represents the 25th percentile, and the upper part represents the 75th percentiles. The whiskers represent the range of the values.

\section{Bacterial load in organs}

The post-mortem quantification of the bacterial load in selected organs revealed high numbers of viable bacteria in the liver, spleen, lungs and kidneys of all studied rabbits (Figures $5 \mathrm{a}-\mathrm{d}$ ). Animals receiving ceftriaxone (either alone or combined with Pep19-2.5) showed lower levels of CFUs in the kidneys and liver (Figures 5a and c, respectively). However, the efficiency of the combined treatment to reduce bacterial colonization in the lungs was lower than that of the ceftriaxone single treatment (Figure 5b). When Pep19-2.5 was administered as a single treatment, it increased the bacterial load in the liver compared with that in untreated animals (Figure 5c) and showed a non-significant trend to do so in the kidneys and lungs (Figures 5a, b and $\mathrm{d}$ ). This phenomenon was not detected in the spleen.

To indirectly quantify the extent of the inflammatory response in animals, we measured the weight of the liver, spleen, lung and kidney of the rabbits. All organs studied remained within the normal weight ranges at the end of the experiment with the exception of the spleen, which showed increased weight in all groups (data not shown).

\section{Histological analysis of the spleen}

The presence of splenomegaly prompted us to perform a more detailed histological study to assess potential differences between the groups. In general, microscopic findings were similarly independent of the treatment, but with different intensity (Supplementary Figure S5). Splenitis was confirmed in samples from all groups and was due to the presence of an inflammatory infiltrate of heterophils (that is, rabbit cells equivalent to polynuclear neutrophils). Abundant bacteria were present in this infiltrate. Bacteria were detected both inside macrophages and free in the extracellular space. Bacterial cells frequently appeared distorted likely because of the activation of immune system effectors (Supplementary Figure S5). The previous findings were accompanied by various degrees of hemorrhage in the splenic tissue. Although there were marked individual responses, in general, treatment with Pep19-2.5 either alone or combined with ceftriaxone, was associated with milder alterations and with a lower bacterial burden than in the untreated controls and in animals that received ceftriaxone single treatment (Table 1).

\section{DISCUSSION}

The current study demonstrated the therapeutic potential of Pep19-2.5, a synthetic peptide, in combination with the antibiotic ceftriaxone in a rabbit model of sepsis. The dual therapy of Pep19-2.5 and ceftriaxone reduced both the bacterial load (Figures 1 and 5) and endotoxin levels in serum (Figure 2), causing a considerable drop in the production of IL-6 (Figure 3a and Supplementary Figure S2A) and TNF $\alpha$ (Figure $3 \mathrm{~b}$ and Supplementary Figure S2B). Moreover, we found an abrogation of hypothermia and a re-establishment of normal rectal temperature (Figure 4), as well as milder alterations and a lower bacterial burden in the spleens of Pep19-2.5/ceftriaxone-treated animals (Table 1).

Despite the intense research and innumerable efforts made in the last decades to develop therapies against sepsis, only modest improvements were introduced in the management of septic patients. ${ }^{16}$ The standard therapy of sepsis comprises an antimicrobial treatment in conjunction with supportive therapies (for example, hemodynamic stabilization and airway management). ${ }^{18}$ In many cases, however, this treatment does not prevent the heavy and fast onset of inflammation characteristic of sepsis. Possible reasons for this failure involve the release of inflammation-inducing toxins (that is, LPS and LP) from the cell envelopes of bacteria triggered by various antibiotics, ${ }^{19}$ which may worsen the patient's pro-inflammatory response. Thus, to be efficient, sepsis therapy must combat the bacterial infection while simultaneously being capable to decrease the inflammatory reaction. To achieve this double objective, we used a classical antibiotic, ceftriaxone, to kill the bacteria and Pep19-2.5 to neutralize endotoxin. 


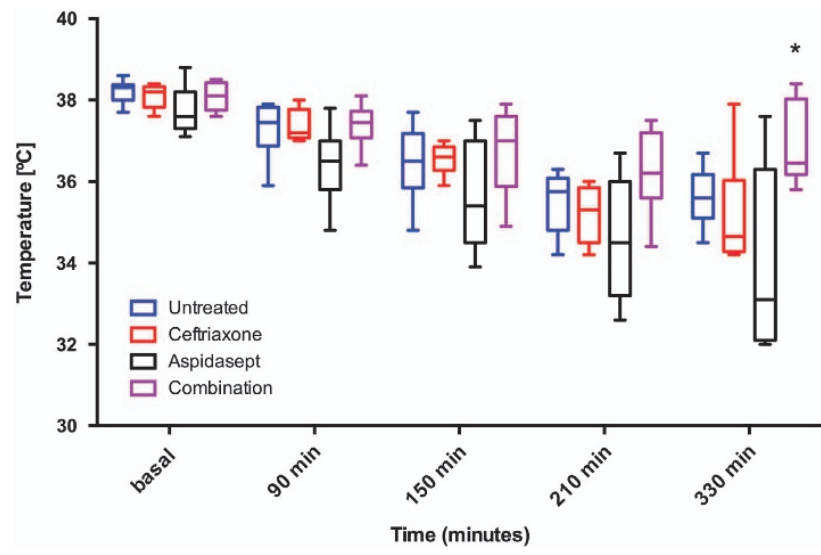

Figure 4 Effect of treatments on rabbit temperature 330 min post challenge. The rectal temperature of each animal group $(n=6)$ was monitored before (designated as 'basal' in the figure) and at a progressively later time points post challenge (until $330 \mathrm{~min}$ ). For group comparisons of the temperatures at different time points, repeated measures factorial design-analysis of variance was performed, followed by Student's $t$-test for related measures, adjusting the average standard deviation. The asterisk indicates the only animal group with a rectal temperature indistinguishable from the control (that is, rabbits receiving the combined treatment, $P<0.05)$. The horizontal line within the boxes represents the median, whereas the lower part represents the 25th percentile, and the upper part represents the 75th percentiles. The whiskers represent the range of the values.

The anti-endotoxic activity of Pep19-2.5 shown in our current work extends previous studies revealing that Pep19-2.5 has LPS-neutralizing capacity in in vitro assays and that this activity is responsible for the survival benefit of endotoxemic mice treated with the peptide. ${ }^{17}$ Using isothermal titration calorimetry, we confirmed the high-affinity binding of Pep19-2.5 to not only purified LPS but also heat-killed Gram-negative and Gram-positive bacterial cells (S. enterica Minnesota and methicillin-resistant Staphylococcus aureus, respectively). ${ }^{20}$ Nevertheless, it is possible that the immunomodulating activity displayed by Pep19-2.5 in the present work is not only due to the peptide's ability to bind and inactivate LPS but also to its inherent capacity to downregulate the inflammatory response. ${ }^{11}$ Using mouse models of endotoxemia and septicemia, we previously demonstrated the therapeutic efficacy of Pep19-2.5 in combination with antibiotics, including ceftriaxone. ${ }^{10}$ In the current study, the rabbit was chosen as the model organism because it is assumed to more closely reflect the response of the human immune system than mice, which are the most commonly used animals in sepsis research. ${ }^{16,21}$ In fact, rabbits are notably less resistant to LPS than mice, although these non-rodents are still far from the exquisite sensitivity of humans to endotoxin. Thus, in current human studies, $2 \mathrm{ng} \mathrm{kg}^{-1}$ of LPS were used to induce endotoxemia; ${ }^{22-24}$ however, in rabbits, a range of $1-10 \mu \mathrm{g} \mathrm{kg}^{-1}$ is applied in i.v. applications. ${ }^{25,26}$ Nevertheless, these acute endotoxemia models do not reflect the progressive course of a real infection and, even less so, the interplay between the different antigenic stimuli and components of the immune
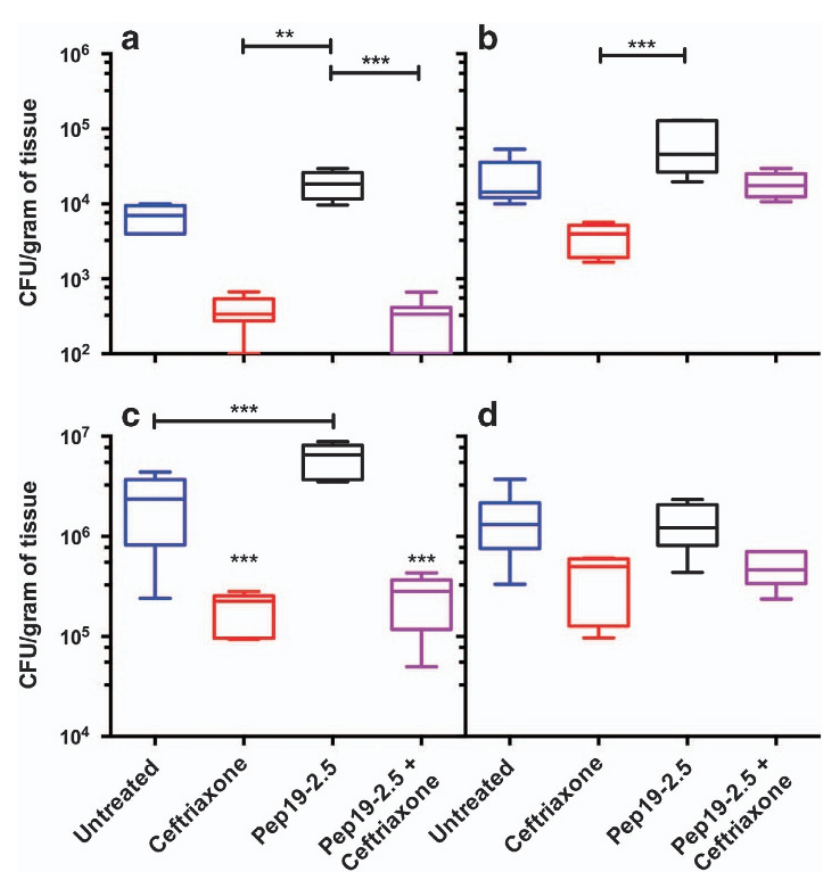

Figure 5 Post-mortem quantification of the bacterial load in selected organs. Post-mortem samples of the kidneys (a), lungs (b), liver (c) and spleen (d) were extracted and weighed. One gram of each sample was homogenized and serially diluted for plating and viable counting. Differences were statistically analyzed using analysis of variance followed by post hoc tests $\left({ }^{*} P<0.05\right.$; $\left.{ }^{* *} P<0.01 ;{ }^{* *} P<0.001\right)$. The horizontal line within the boxes represents the median, whereas the lower part represents the 25th percentile, and the upper part represents the 75th percentiles. The whiskers represent the range of the values.

system that is characteristic of an infectious process. By contrast, surgical models, such as cecal ligation and puncture, do reflect the pathophysiological features of human sepsis; however, these models use uncharacterized mixtures of bacterial populations as the endotoxic stimulus, thereby hindering the intra-assay and inter-assay reproducibility. ${ }^{27}$ For these reasons, sepsis was induced in our model by the i.v. inoculation of live S. enterica cells to resemble an infectious process, while simultaneously ensuring the necessary reproducibility. Furthermore, the induction of bacteremia in our model is of importance because, in one-third of human sepsis cases, it is possible to obtain a blood-positive culture. ${ }^{28}$ Finally, patients with blood-positive cultures have a higher risk of experiencing cardiovascular events (for example, myocardial infarction) even years after infection. ${ }^{29}$

Similar to our findings, other authors using different rabbit models of sepsis reported metabolic disorders such as hyperglycemia and hyperlactatemia, ${ }^{30,31}$ and showed that the experimental treatment reduced lactate levels. However, in these studies, animals received therapy before being infected ${ }^{30}$ or continuous infusion of the agent, ${ }^{31}$ unlike in our study. Similarly, Garcia et al. ${ }^{32}$ reported amelioration of acidosis in a rabbit model in which animals were pre-treated with the experimental drug. 
Table 1 Summary of anatomopathological findings in the histological preparations of the rabbit spleen ${ }^{\mathrm{a}}$

\begin{tabular}{|c|c|c|c|c|c|}
\hline Sample from the indicated animal group & Hemorrhage & Degree of necrosis & Degree of splenitis & Size of follicles & Number of bacteria \\
\hline Untreated 1 & +++++ & ++++ & ++++ & Reduced & +++++ \\
\hline Untreated 2 & +++ & ++++ & ++++ & Reduced & +++++ \\
\hline Untreated 4 & ++++ & +++ & ++++ & Normal & +++ \\
\hline Untreated 5 & +++ & ++++ & ++++ & Normal & +++ \\
\hline Ceftriaxone 1 & ++ & +++ & +++ & Normal & ++ \\
\hline Ceftriaxone 2 & ++++ & +++ & +++ & Normal & ++ \\
\hline Ceftriaxone 3 & ++++ & ++ & ++ & Normal & + \\
\hline Ceftriaxone 4 & ++++ & +++ & ++++ & Normal & ++ \\
\hline Ceftriaxone 5 & +++ & ++++ & ++++ & Normal & +++ \\
\hline Pep19-2.5 3 & ++ & + & +++ & Normal & ++ \\
\hline Pep19-2.5 4 & ++ & + & ++ & Increased & ++ \\
\hline Pep19-2.5 5 & +++ & + & ++ & Normal & + \\
\hline Pep19-2.5 6 & ++ & + & ++ & Normal & + \\
\hline Pep19-2.5+ceftriaxone 1 & ++ & ++ & +++ & Normal & +++ \\
\hline Pep19-2.5+ceftriaxone 2 & + & + & ++ & Normal & + \\
\hline Pep19-2.5+ceftriaxone 3 & ++ & + & + & Reduced & + \\
\hline Pep19-2.5+ceftriaxone 4 & + & + & + & Normal & + \\
\hline Pep19-2.5+ceftriaxone 5 & ++ & ++ & ++ & Normal & + \\
\hline Pep19-2.5+ceftriaxone 6 & ++ & + & ++ & Normal & + \\
\hline
\end{tabular}

Anatomopathological evaluation of all samples was carried out in a blinded manner by the same pathologist, according to the institutional guidelines.

apost-mortem samples of the spleen were fixed by immersion in $4 \%$ formaldehyde and were subsequently prepared for inclusion, cutting and hematoxylin-eosin staining.

The release of endotoxin from antibiotic-treated bacteria ${ }^{33}$ is well documented both in vitro ${ }^{34}$ and during active infection in mice, ${ }^{35}$ rats $^{36}$ and rabbits. ${ }^{30}$ The administration of AMPs alone or combined with antibiotics has been reported to reduce the LPS levels and counteract sepsis-associated symptoms, leading to survival benefits in several animal models, including mice and rats. ${ }^{37-40}$ Using rabbit models, other authors have reported that polymyxin B alleviated pathophysiological disorders caused by endotoxemia ${ }^{41}$ or septicemia. ${ }^{42,43}$ Similarly, Lin et al. ${ }^{30}$ showed that a combination of an AMP (a fragment of the bactericidal/permeability-increasing protein (rBPI21)) and cefamandole accelerated bacterial clearance, improved cardiopulmonary dysfunction and prevented rabbit death; however, in this model, the antibiotic treatment was given before bacterial challenge. Finally, other groups reported that a recombinant endotoxin neutralizing protein from Limulus polyphemus combined with antibiotics partially protected rabbits from lethal peritonitis ${ }^{44,45}$ or from lethal endotoxemia if animals received antiendotoxic therapy before being infected. ${ }^{32}$

To the best of our knowledge, this is the first report showing evidence in rabbits of AMP-mediated inactivation of endotoxin (Figure 2), leading not only to the neutralization of pro-inflammatory cytokine production (Figure 3 ) but also to the recovery of normal temperature values (Figure 4). Our results suggest that Pep19-2.5 and ceftriaxone act in synergy to combat bacteremia (Figure 1) and neutralize IL-6 production (Figure 3). This phenomenon is particularly relevant because any anti-sepsis drug candidate is expected to be administered in combination with one or several antibiotics. Keeping in mind that we administered the drugs as a bolus, it is likely that a constant infusion might result in an even more pronounced therapeutic effect. Taken together, our results indicate that the therapy of Pep19-2.5 and ceftriaxone is a promising drug combination for controlling the crucial causes of sepsis.

\section{CONFLICT OF INTEREST}

The authors declare no conflict of interest.

\section{ACKNOWLEDGEMENTS}

We are indebted to Ismael Aizpún Ayesa (Department of Pharmacology and Toxicology, University of Navarra) for his excellent technical assistance in the animal experiments. The gift of the HEK-293/TLR4 and HEK-293/LacZ cell lines by Dr Juan José Lasarte is greatly appreciated. GMT was funded by grants from Proyectos de Investigacion Universidad de Navarra (PIUNA-P2011-17 and P2015-14), Spain. SBV is recipient of a doctoral fellowship from Friends of the University of Navarra, Spain. Pep19-2.5 patent OCT/ 
EP2009/002565. Research at the laboratory of LH is supported by the Deutsche Forschungsgemeinschaft (DFG, project DR797/3-1 611672). $\mathrm{KB}, \mathrm{LH}, \mathrm{TS}, \mathrm{SF}$ and GMT are members of the spin-off company 'Brandenburg Antiinfektiva GmbH' (www.antiinfektiva.com) from the Borstel Research Institute.

1 Singer M, Deutschman CS, Seymour CW, Shankar-Hari M, Annane D, Bauer $M$ et al. The third international consensus definitions for sepsis and septic shock (Sepsis-3). JAMA 2016; 315: 801-810.

2 Kempker JA, Martin GS. The changing epidemiology and definitions of sepsis. Clin Chest Med 2016; 37: 165-179.

3 Rittirsch D, Hoesel LM, Ward PA. The disconnect between animal models of sepsis and human sepsis. J Leukocyte Biol 2007; 81: 137-143.

4 Lakshmikanth CL, Jacob SP, Chaithra VH, de Castro-Faria-Neto HC, Marathe GK. Sepsis: in search of cure. Inflamm Res 2016; 65: 587-602.

5 Poli-de-Figueiredo LF, Garrido AG, Nakagawa N, Sannomiya P. Experimental models of sepsis and their clinical relevance. Shock 2008; 30(Suppl 1): 53-59.

6 Evans ME, Pollack M. Effect of antibiotic class and concentration on the release of lipopolysaccharide from Escherichia coli. J Infect Dis 1993; 167: 1336-1343.

7 Silverstein R, Wood JG, Xue Q, Norimatsu M, Horn DL, Morrison DC. Differential host inflammatory responses to viable versus antibiotic-killed bacteria in experimental microbial sepsis. Infect Immun 2000; 68: 2301-2308.

8 Arbeit RD, Maki D, Tally FP, Campanaro E, Eisenstein BIDaptomycin 98-01 and 99-01 Investigators, The safety and efficacy of daptomycin for the treatment of complicated skin and skin-structure infections. Clin Infect Dis 2004; 38: 1673-1681.

9 Martinez de Tejada G, Sanchez-Gomez S, Razquin-Olazaran I, Kowalski I, Kaconis $Y$, Heinbockel $L$ et al. Bacterial cell wall compounds as promising targets of antimicrobial agents I. Antimicrobial peptides and lipopolyamines. Curr Drug Targets 2012; 13: 1121-1130.

10 Heinbockel L, Sanchez-Gomez S, Martinez de Tejada G, Domming S, Brandenburg J, Kaconis $Y$ et al. Preclinical investigations reveal the broad-spectrum neutralizing activity of peptide Pep19-2.5 on bacterial pathogenicity factors. Antimicrob Agents Chemother 2013; 57: $1480-1487$.

11 Schuerholz T, Doemming S, Hornef M, Martin L, Simon TP, Heinbockel L et al. The anti-inflammatory effect of the synthetic antimicrobial peptide 19-2.5 in a murine sepsis model: a prospective randomized study. Crit Care 2013; 17: R3.

12 Nemzek JA, Hugunin KM, Opp MR. Modeling sepsis in the laboratory: merging sound science with animal well-being. Comp Med 2008; 58: $120-128$

13 Le Roy D, Di Padova F, Adachi Y, Glauser MP, Calandra T, Heumann D. Critical role of lipopolysaccharide-binding protein and CD14 in immune responses against gram-negative bacteria. J Immunol 2001; 167: 2759-2765.

14 Galanos C, Freudenberg MA. Mechanisms of endotoxin shock and endotoxin hypersensitivity. Immunobiology 1993; 187: 346-356.

15 Taveira da Silva AM, Kaulbach HC, Chuidian FS, Lambert DR, Suffredini AF, Danner RL. Brief report: shock and multiple-organ dysfunction after self-administration of Salmonella endotoxin. $N$ Eng/ J Med 1993; 328: 1457-1460.

16 Fink MP. Animal models of sepsis. Virulence 2014; 5: 143-153.

17 Gutsmann T, Razquin-Olazaran I, Kowalski I, Kaconis Y, Howe J, Bartels R et al. New antiseptic peptides to protect against endotoxin-mediated shock. Antimicrob Agents Chemother 2010; 54: 3817-3824.

18 Dellinger RP, Levy MM, Rhodes A, Annane D, Gerlach H, Opal SM et al. Surviving sepsis campaign: international guidelines for management of severe sepsis and septic shock: 2012. Crit Care Med 2013; 41: 580-637.

19 Morrison DC, Bucklin SE. Evidence for antibiotic-mediated endotoxin release as a contributing factor to lethality in experimental gram-negative sepsis. Scand J Infect Dis Supp/ 1996; 101: 3-8.

20 Martinez de Tejada G, Heinbockel L, Ferrer-Espada R, Heine H, Alexander $\mathrm{C}$, Barcena-Varela $\mathrm{S}$ et al. Lipoproteins/peptides are sepsis-inducing toxins from bacteria that can be neutralized by synthetic anti-endotoxin peptides. Sci Rep 2015; 5: 14292.
21 Parker SJ, Watkins PE. Experimental models of gram-negative sepsis. Br J Surg 2001; 88: 22-30.

22 Schoergenhofer C, Schwameis M, Hobl EL, Ay C, Key NS, Derhaschnig U et al. Potent irreversible P2Y12 inhibition does not reduce LPS-induced coagulation activation in a randomized, double-blind, placebocontrolled trial. Clin Sci 2016; 130: 433-440.

23 Doorduin J, Leentjens J, Kox M, van Hees HW, van der Hoeven JG, Pickkers $\mathrm{P}$ et al. Effects of experimental human endotoxemia on diaphragm function. Shock 2015; 44: 316-322.

24 Kiers D, Gerretsen J, Janssen E, John A, Groeneveld R, van der Hoeven JG et al. Short-term hyperoxia does not exert immunologic effects during experimental murine and human endotoxemia. Sci Rep 2015; 5: 17441.

25 Krishnamurti C, Carter AJ, Maglasang P, Hess JR, Cutting MA, Alving BM. Cardiovascular toxicity of human cross-linked hemoglobin in a rabbit endotoxemia model. Crit Care Med 1997; 25: 1874-1880.

26 Fink MP, Morrissey PE, Stein KL, Clement RE, Fiallo V, Gardiner WM. Systemic and regional hemodynamic effects of cyclo-oxygenase and thromboxane synthetase inhibition in normal and hyperdynamic endotoxemic rabbits. Circ Shock 1988; 26: 41-57.

27 Wichtermann KA, Bane AE, Chandry IA. Sepsis and septic shock - a review of laboratory models and a proposal. J Surg Res 1980; 29: 189-201.

28 Ranieri VM, Thompson BT, Barie PS, Dhainaut JF, Douglas IS, Finfer S et al. Drotrecogin alfa (activated) in adults with septic shock. N Engl J Med 2012; 366: 2055-2064.

29 Jafarzadeh SR, Thomas BS, Marschall J, Fraser VJ, Gill J, Warren DK. Quantifying the improvement in sepsis diagnosis, documentation, and coding: the marginal causal effect of year of hospitalization on sepsis diagnosis. Ann Epidemiol 2016; 26: 66-70.

30 Lin Y, Leach WJ, Ammons WS. Synergistic effect of a recombinant $\mathrm{N}$-terminal fragment of bactericidal/permeability-increasing protein and cefamandole in treatment of rabbit gram-negative sepsis. Antimicrob Agents Chemother 1996; 40: 65-69.

31 Camerota AJ, Creasey AA, Patla V, Larkin VA, Fink MP. Delayed treatment with recombinant human tissue factor pathway inhibitor improves survival in rabbits with gram-negative peritonitis. J Infect Dis 1998; 177: 668-676.

32 Garcia C, Saladino R, Thompson C, Hammer B, Parsonnet J, Wainwright N et al. Effect of a recombinant endotoxin-neutralizing protein on endotoxin shock in rabbits. Crit Care Med 1994; 22: 1211-1218.

$33 \mathrm{Nau} \mathrm{R}$, Eiffert H. Modulation of release of proinflammatory bacterial compounds by antibacterials: potential impact on course of inflammation and outcome in sepsis and meningitis. Clin Microbiol Rev 2002; 15: 95-110.

34 Cohen J, McConnell JS. Release of endotoxin from bacteria exposed to ciprofloxacin and its prevention with polymyxin B. Eur J Clin Microbiol 1986; 5: 13-17.

35 Sawa T, Kurahashi K, Ohara M, Gropper MA, Doshi V, Larrick JW et al. Evaluation of antimicrobial and lipopolysaccharide-neutralizing effects of a synthetic CAP18 fragment against Pseudomonas aeruginosa in a mouse model. Antimicrob Agents Chemother 1998; 42: 3269-3275.

36 Giacometti A, Cirioni O, Ghiselli R, Mocchegiani F, Paggi AM, Orlando F et al. Therapeutic efficacy of intraperitoneal polymyxin $B$ and polymyxinlike peptides alone or combined with levofloxacin in rat models of septic shock. J Antimicrob Chemother 2002; 49: 193-196.

37 Ghiselli R, Giacometti A, Cirioni O, Mocchegiani F, Viticchi C, Scalise $\mathrm{G}$ et al. Cationic peptides combined with betalactams reduce mortality from peritonitis in experimental rat model. J Surg Res 2002; 108: 107-111.

38 Giacometti A, Cirioni O, Ghiselli R, Mocchegiani F, Viticchi C, Orlando F et al. Antiendotoxin activity of protegrin analog IB-367 alone or in combination with piperacillin in different animal models of septic shock. Peptides 2003; 24: 1747-1752.

39 Fukumoto K, Nagaoka I, Yamataka A, Kobayashi H, Yanai T, Kato Y et al. Effect of antibacterial cathelicidin peptide CAP18/LL-37 on sepsis in neonatal rats. Pediatr Surg Int 2005; 21: 20-24.

40 Murakami T, Obata T, Kuwahara-Arai K, Tamura H, Hiramatsu K, Nagaoka I. Antimicrobial cathelicidin polypeptide CAP11 suppresses the production and release of septic mediators in D-galactosamine-sensitized endotoxin shock mice. Int Immunol 2009; 21: 905-912.

41 Corrigan JJ Jr, Bell BM. Comparison between the polymyxins and gentamicin in preventing endotoxin-induced intravascular coagulation and leukopenia. Infect Immun 1971; 4: 563-566. 
42 Corrigan JJ Jr., Kiernat JF. Effect of polymyxin B sulfate on endotoxin activity in a gram-negative septicemia model. Pediatr Res 1979; 13: 48-51.

43 Flynn PM, Shenep JL, Stokes DC, Fairclough D, Hildner WK. Polymyxin B moderates acidosis and hypotension in established, experimental gram-negative septicemia. J Infect Dis 1987; 156: 706-712.

44 Saladino R, Garcia C, Thompson C, Hammer B, Parsonnet J, Novitsky T et al. Efficacy of a recombinant endotoxin neutralizing protein in rabbits with Escherichia coli sepsis. Circ Shock 1994; 42: 104-110.

45 Saladino RA, Stack AM, Thompson C, Sattler F, Novitsky TJ, Siber GR et al. High-dose recombinant endotoxin neutralizing protein improves survival in rabbits, with Escherichia coli sepsis. Crit Care Med 1996; 24: 1203-1207. (c) (i) $(9)$

This work is licensed under a Creative Commons Attribution-NonCommercial-NoDerivs 4.0 International License. The images or other third party material in this article are included in the article's Creative Commons license, unless indicated otherwise in the credit line; if the material is not included under the Creative Commons license, users will need to obtain permission from the license holder to reproduce the material. To view a copy of this license, visit http://creativecommons.org/licenses/by-nc-nd/4.0/

Supplementary Information accompanies the paper on Experimental \& Molecular Medicine website (http://www.nature.com/emm) 